\title{
Exploring Heterocycle-Spermine Conjugates as Modulators of Oncogenic microRNAs Biogenesis
}

Cécile Becquart, ${ }^{\dagger}$ Myriame Le Roch, ${ }^{\dagger}$ Stéphane Azoulay, ${ }^{\dagger}$ Philippe Uriac, ${ }^{\ddagger}$ Audrey Di Giorgio, ${ }^{\dagger}$ and Maria Duca*,†

${ }^{\dagger}$ Université Côte d'Azur, CNRS, Institute of Chemistry of Nice (ICN), Nice 06100, France

${ }^{\ddagger}$ Université de Rennes 1, CNRS UMR6226, Institut of Chemistry of Rennes (ISCR), Rennes 35042, France

\section{Supporting Information}

ABSTRACT: Targeting biologically relevant noncoding RNAs using small molecules is currently one of the major challenges of medicinal chemistry but holds a great potential for future therapeutic applications. In this context, oncogenic microRNAs represent a particularly promising target, and various examples of RNA binders have been reported as inhibitors of the biogenesis of these microRNAs. Here, we report the biochemical evaluation and molecular docking studies of five heterocycle-spermine conjugates revealing that a structure-based design of efficient and especially selective inhibitors can be performed based on the secondary and tertiary configuration of the targeted RNA.

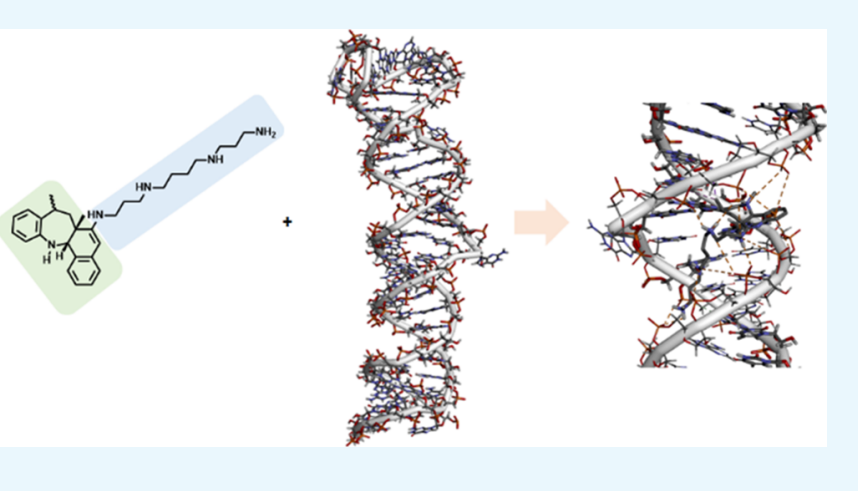

\section{INTRODUCTION}

RNA has long been considered an intermediate in the transmission of the genetic information from the genotype to the phenotype, but during the last decades, its pivotal role in almost every biological process, especially in the tight regulation of gene expression, led to the recognition of its capital importance for cellular homeostasis and survival. ${ }^{1}$ Although coding RNAs are essential for protein expression, being first transcribed from DNA and then translated into proteins, a large number of noncoding RNAs having complex and essential functions in almost every intracellular event have been and continue to be identified. ${ }^{2}$ MicroRNAs are a class of these noncoding RNAs that are involved in the regulation of gene expression. ${ }^{3}$ These short (22-25 nucleotides) RNA oligonucleotides recognize particular mRNA sequences and inhibit the translation of the corresponding protein, thus performing a tight control of protein expression. Each miRNA is able to target hundreds of mRNAs explaining the capital role of these short RNAs in cellular homeostasis. ${ }^{4}$ miRNAs are transcribed from a miRNA gene in the nucleus to form a primary miRNA (pri-miRNA) of about $1 \mathrm{~kb} .{ }^{5}$ Each pri-miRNA is then processed by a nuclear ribonuclease called Drosha, leading to the formation of a shorter miRNA precursor (premiRNA) of about 70 nucleotides. The pre-miRNA is then moved to the cytoplasm, where it is further processed by another ribonuclease called Dicer into a shorter RNA duplex. After unwinding, the mature miRNA forms a multiproteic complex called miRISC, which is eventually responsible for the recognition of one or more mRNAs, leading to the degradation and to the above-mentioned inhibition of translation. ${ }^{6}$
Whereas miRNAs biogenesis and function are essential for cell survival, the deregulation in the expression of miRNAs, such as the overexpression or the underexpression of some of them, has been directly linked to a wide number of pathologies such as cancer. ${ }^{7}$ miRNAs thus became extremely attractive therapeutic targets either for the inhibition of the overexpressed ones and the activation of the expression of the underexpressed miRNAs. ${ }^{8}$ Oligonucleotides have obviously been the first approach employed with this aim because they can act both as antisense against intracellular miRNAs, thus inhibiting their function, or supply for lacking miRNAs. ${ }^{9}$ These agents showed to be very specific and very efficient during in vitro studies, but their therapeutic applications remain poor due to unfavorable pharmacodynamics and pharmacokinetic properties. ${ }^{8}$ For this reason, the possibility of using small molecules against miRNAs rapidly emerged as a promising approach and currently represents an important challenge for medicinal chemistry. ${ }^{10}$ Because the targeting of short singlestranded RNAs using small molecules is not feasible, researchers in the field focused their attention on the targeting of miRNAs precursors such as pri-miRNAs and pre-miRNAs. Both bear a stem-loop structure containing various internal loops and bulges due to the presence of double-stranded regions associated with single-stranded ones. These stem-loopstructured RNAs adopt particular three-dimensional (3D) conformations, thus resembling much more a protein than a double-helical DNA. ${ }^{11}$ Their three-dimensional structure is

Received: October 7, 2018

Accepted: November 22, 2018

Published: December 4, 2018 
A<smiles></smiles>

B

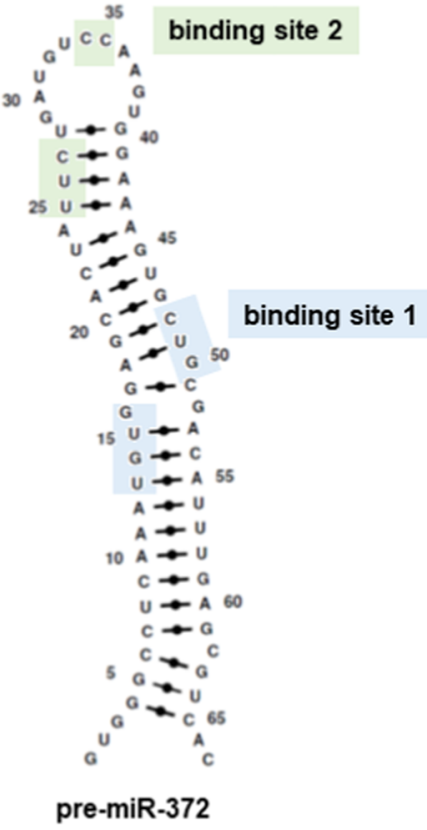

C
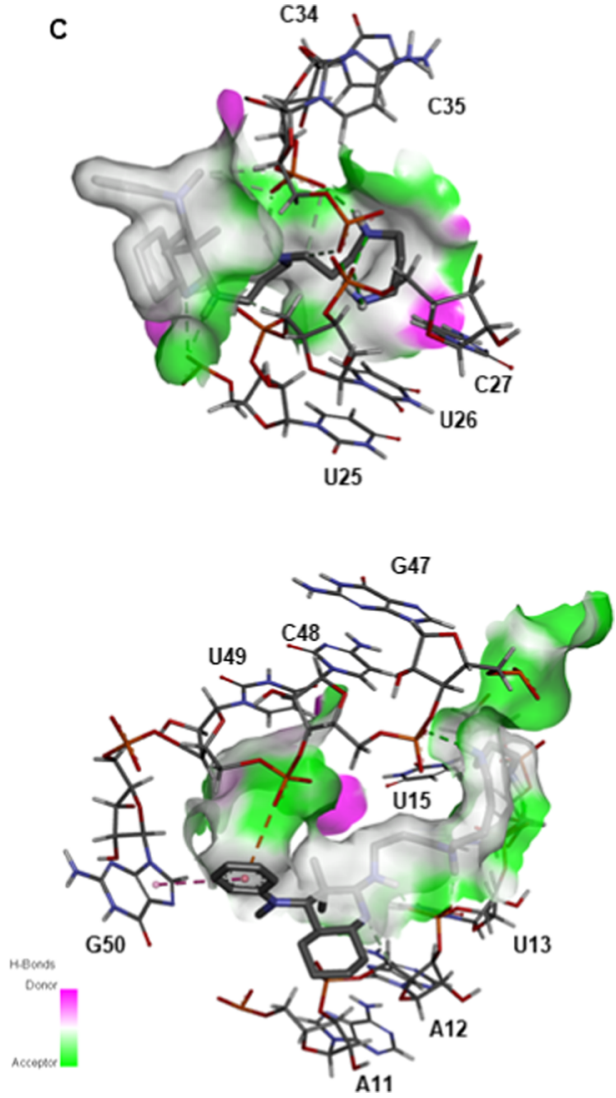

Figure 1. (A) Chemical structure of conjugated polyamine PA-1 previously identified as an inhibitor of pre-miR-372 Dicer-mediated processing. (B) Primary and secondary structures of pre-miR-372, where the two possible binding sites of PA-1 are underlined in blue and green. (C) PA-1 interaction with pre-miR-372 as suggested by molecular docking studies performed using Autodock. H-bonds donor-acceptor surfaces on the RNA are represented in pink and green, respectively.

druggable using natural and synthetic compounds, as demonstrated by a number of recent works reporting in particular the inhibition of miRNAs biogenesis using smallmolecule compounds with various selectivity profiles. ${ }^{12-21}$ Various approaches have been applied to the discovery of small molecules able to target miRNA biogenesis with efficacy and specificity in an intracellular context. ${ }^{9,11,22-24}$ These approaches include screening of large compound libraries against the widely distributed oncogenic miR-21, ${ }^{14}$ screening of focused libraries containing compounds known to interact with structured $\mathrm{RNAs}^{18,25}$ and more advanced screening techniques including Inforna, ${ }^{17}$ which is based on the combination of library-vs-library two-dimensional combinatorial screening (2-DCS $)^{26}$ and a statistical method to score the binding interactions called StARTS. ${ }^{27}$

In our recent works, we focused on the search for RNA binders using both the design of multimodal RNA ligands and library screening. In particular, we looked for compounds that could decrease the intracellular levels of miR-372, which is oncogenic in several types of cancers and particularly involved in the proliferation of gastric adenocarcinoma cells (AGS cells) ${ }^{18-20}$ MiR-372 shares with miR-373 the seed sequence (the sequence that recognizes the target mRNA). Both miR372 and miR-373 originate from a unique cluster in the genome $^{28}$ and are expressed in some gastric tumors, but they are undetectable in normal gastric epithelial cells. Overexpression of miR-372 represses the translation of the tumor suppressor protein LATS2 in these cancers. ${ }^{29}$ In this context, we recently performed the screening of a 640-compound library and the identification of polyamine derivatives interfering with in vitro Dicer-mediated processing of the oncogenic miR-372 precursor (pre-miR-372). ${ }^{20}$ This led to the identification of functionalized polyamines as efficient RNA binders and inhibitors of miRNA biogenesis. The most active molecule, PA-1 (Figure 1A), ${ }^{30}$ was able to bind pre-miR-372 and inhibit its processing in vitro. A dose-dependent and -specific inhibition of AGS proliferation concomitantly with a decrease in miR-372 amount and derepression of LATS2 expression demonstrated that functionalized polyamines are efficient and selective miRNAs interfering agents in vitro and in cells. Furthermore, the obtained results suggested that further improvement of binding affinity and selectivity was possible upon modification of the heterocyclic moiety, thus leading to more efficient and specific interactions and a better fit of the ligand in the RNA-binding pocket.

In this work, we report the biophysical and biochemical evaluation of polyamine analogues of PA-1 chosen on the basis of molecular docking of PA-1/pre-miR-372 complex. In fact, these theoretical measurements, combined with experimental footprinting, suggested that the binding of PA-1 to its target was mainly governed by hydrogen bonds and electrostatic interactions with the phosphate backbone at the level of residues A12-U15 and C48-G50 internal loop (Figure $1 \mathrm{~B}, \mathrm{C}){ }^{20} \mathrm{~A}$ second site of interaction was possibly close to the stem-loop junction at residues $\mathrm{U} 25-\mathrm{C} 27$ and $\mathrm{C} 34-\mathrm{C} 35$ and based on similar interactions. Binding was selective because PA-1 was able to bind and affect the processing of only a small set of miRNAs in vitro and in cells leading to a 
<smiles>CNc1ccc(C2c3ccccc3N=C(NCCCNCCCCNCCCN)C2(C)C)cc1</smiles><smiles>CC(C)[C@]12c3ccccc3N=C(NCCCNCCCCNCCCN)[C@@]1(C)C[C@H](C)c1ccccc1N2C</smiles><smiles>NCCCNCCCCNCCCNC1=Nc2ccccc2CCC1</smiles><smiles>NCCCNCCCCNCCCNC1=Nc2ccccc2CC1</smiles><smiles>NCCCNCCCCNCCCN</smiles><smiles>C[C@@H]1C[C@]2(C)C(=S)Nc3ccccc3[C@@]2(C)N(C)c2ccccc21</smiles><smiles>c1ccc2ncccc2c1</smiles>

spermine benzazepinoquinoline

quinoline

Figure 2. Chemical structures of heterocycle-spermine conjugates PA-2 to PA-5 chosen for the study.

very specific biological effect, suggesting that other functionalized polyamines could lead to even better activities. Based on these observations, we selected four analogues of PA-1, namely, PA-2 to PA-5 (Figure 2), which we have previously synthesized for the study of polyamine transportation system. ${ }^{30}$ These compounds are conjugates of spermine to various heterocyclic moieties and have been employed to evaluate the structure-activity relationships of this kind of functionalized polyamines. We thus studied the affinity and the selectivity of all the compounds for pre-miR-372 as well as the inhibition activity on Dicer-mediated processing of this pre-miRNA. Comparison of the obtained results with the ones gathered on other pre-miRNA sequences led to the identification of PA-3 as the most efficient inhibitor of pre-miR-372 processing both in terms of activity and selectivity. The detailed study of the molecular mechanism of interaction with the targeted RNA further highlights the potential of functionalized polyamines for the future design of more efficient and specific inhibitors.

\section{RESULTS AND DISCUSSION}

Choice of Heterocycle-Spermine Conjugates for Structure-Affinity and Structure-Activity Studies. Binding studies performed on functionalized polyamine PA-1 revealed that most of the interactions formed with pre-miR372 involved phosphate groups of the RNA backbone located close to the stem-bulge and the stem-loop junction (Figure 1B). ${ }^{20}$ Furthermore, the role of the 3,4-dihydroquinoline scaffold in the interaction with the target was minor, with a $\pi-\pi$ interaction with the G50 base and $\pi$-anion interaction with the adjacent phosphate group, whereas the polyamine chain groups dominated the binding. A closer look at the binding pocket of PA-1 also revealed that the 3,4dihydroquinoline moiety did not suitably fit in the binding pocket formed by RNA residues in any of the possible binding sites, suggesting that binding could be improved upon major involvement of this moiety and upon formation of more specific interactions such as hydrogen bonds with paired and nonpaired nucleobases. To confirm this hypothesis, we decided to study the in vitro activity of various analogues of PA-1 (Figure 2). First, PA-2 containing the $N$-methylbenzenamine substituent in position 4 of the 3,4-dihydroquinoline was chosen due to its similarity to PA-1 and for the different distribution of heteroaromatic rings and replacement of the tertiary amine by a secondary one with respect to the parent compound. Second, a constrained analogue of PA-1 containing a 3,4-dihydroquinoline ring fused with a cis junction to 2,3,4,5tetrahydro-1H-benzazepine (PA-3) was employed. In parallel, analogues bearing smaller heterocyclic substituents such as 2,3,4,5-tetrahydro-1H-benzazepine (PA-4) and 3,4-dihydroquinoline (PA-5) were evaluated. Finally, and to measure the role of each moiety, the polyamine spermine, the benzazepinoquinoline tetracycle, and quinoline were employed as controls (Figure 2).

The aim of the study of this set of compounds is to afford a better understanding of the potential interactions formed by heterocycle-spermine conjugates toward the design of more selective and stronger inhibitors of miRNAs biogenesis.

Evaluation of the Inhibition Activity of HeterocycleSpermine Conjugates on pre-miR-372. Analogous to what we performed in a previous study about functionalized polyamines, ${ }^{20}$ in this study, we focused on miR-372, which is the most abundant oncogenic miRNA in gastric adenocarcinoma AGS cells and whose expression is strongly linked to cellular proliferation.

We previously reported that PA-1 inhibited Dicer-mediated processing of pre-miR-372 in vitro with $\mathrm{IC}_{50}$ of $1.06 \mu \mathrm{M}^{20}$ For this kind of measurement, we employ a cell-free assay based on the fluorescence resonance energy transfer (FRET) technique in which the targeted pre-miRNA is double labeled with a fluorophore (fluorescein or FAM) and a quencher (dabcyl or $\mathrm{DAB})$ at $3^{\prime}$ and $5^{\prime}$ ends, respectively. ${ }^{18}$ In the presence of Dicer enzyme, this latter cleaves the RNA and appearance of 
fluorescence is observed. If a RNA ligand strongly binds to the structured pre-miRNA and inhibits the cleavage by Dicer, no fluorescence is detected. This assay has been previously validated and demonstrated to be highly reliable for the identification of the most active compounds in subsequent intracellular studies. ${ }^{18-20}$ Performing this assay over a range of inhibitor concentrations allows for the appreciation of $\mathrm{IC}_{50}$ values (Figure S1 in the Supporting Information). We thus evaluated the in vitro activity of compounds PA-1 to PA-5 and the corresponding results are reported in Table 1. PA-2, which

Table 1. Inhibition Activities $\left(\mathrm{IC}_{50}, \mu \mathrm{M}\right)$ for the Inhibition of pre-miR-372 Dicer-Mediated Processing in the Presence of Compounds PA-1/PA-5

$\begin{array}{cccc}\mathrm{ID} & \mathrm{IC}_{50}{ }^{a} \text { recombinant Dicer } & \mathrm{IC}_{50}{ }^{\prime a} \mathrm{MCF}-7 \text { lysates } & \mathrm{IC}_{50}{ }^{\prime} / \mathrm{IC}_{50} \\ \text { PA-1 } & 1.06 \pm 0.09 & 20.8 \pm 1.2 & 20 \\ \text { PA-2 } & 0.315 \pm 0.003 & 7.91 \pm 0.9 & 25 \\ \text { PA-3 } & 0.580 \pm 0.01 & 9.84 \pm 1.1 & 17 \\ \text { PA-4 } & 10.0 \pm 2.1 & >50 & \\ \text { PA-5 } & \text { no activity } & \text { no activity } & \end{array}$

${ }^{a} \mathrm{IC}_{50}$ experiments were performed in the presence of $50 \mathrm{nM}$ of $3^{\prime}$ dabcyl-5'-FAM-pre-miR-372 beacon and $0.25 \mathrm{U}$ of human recombinant Dicer in buffer A $\left(\mathrm{IC}_{50}\right)$ or $250 \mu \mathrm{g} / \mathrm{mL}$ of MCF-7 lysates $\left(\mathrm{IC}_{50}{ }^{\prime}\right)$ and represent the average of two independent experiments with their standard deviation.

bears a structure close to PA-1, showed a 3-fold improved activity $\left(\mathrm{IC}_{50}=0.315\right.$ vs $\left.1.06 \mu \mathrm{M}\right)$. PA-3 showed a similar improvement in the activity $\left(\mathrm{IC}_{50}=0.580 \mu \mathrm{M}\right)$ compared to PA-1 and together with PA-2 was the best inhibitor. PA-4 retained some activity but with a 10 -fold loss compared to PA1. Finally, PA-5, which does not contain the supplementary methylaminophenyl substituent of PA-1 and PA-2 completely lost activity. These results show that slight modifications in the spatial distribution of these polyamines induces changes in the mode of interaction and inhibition efficacy. The methylaminophenyl substituent was clearly involved in the interaction of PA-1 with the target, explaining why its removal in PA-5 leads to a loss of activity. Furthermore, these results confirm that more extended aromatic substituents of PA-1, PA-2, and PA-3 lead to better activities. Noteworthy, spermine, quinoline, and the benzazepinoquinoline derivative taken as controls did not show any activity at the tested concentrations, suggesting that the spermine side chain and the heterocyclic substituent are both essential for the efficient inhibition of Dicer processing.

To understand if the observed inhibition of pre-miR-372 cleavage was selective, all the compounds were then evaluated for their ability to inhibit pre-miR-372 processing in the presence of MCF-7 lysates instead of human recombinant Dicer. In fact, cell lysates contain large amounts of potential competitors for the binding of RNA ligands and their use in cell-free assays puts the compounds in conditions that are closer to those found in intracellular assays. The obtained results are eventually more relevant. We tested compounds PA-1, PA-2, and PA-5 in the presence of MCF-7 lysates instead of human recombinant Dicer. Results are reported in Table 1 and show that the inhibition effect is lower by 20 times for PA-1, 25 times for PA-2, and 17 times for PA-3, whereas the $\mathrm{IC}_{50}$ values for PA-4 and PA-5 could not be measured at the tested concentrations. Thus, the inhibition activity is conserved in the presence of lysates, PA-3 being the most selective compound, even if compounds are not completely specific.

Evaluation of Compounds PA-1 to PA-5 for pre-miR372 Binding Affinity and Selectivity. The experiments described so far do not show if the studied polyamines inhibit Dicer processing upon pre-miR-372 binding or after direct, and thus not specific, inhibition of Dicer. To evaluate the binding of compounds PA-1 to PA-5 for pre-miR-372, we thus measured the dissociation constants $\left(K_{\mathrm{D}}\right)$ for this target using a monolabeled pre-miR-372 fragment bearing a fluorescein fluorophore at the 5 -end in the absence of Dicer enzyme. The evaluation of fluorescence variation as a function of the concentration of compounds allows for the measurement of $K_{\mathrm{D}}$ values (Table 2 and Figure S2 in the Supporting Information). ${ }^{18}$ The obtained results showed that all the compounds bore sub-micromolar affinities, with $K_{\mathrm{D}}$ values ranging from 0.11 to $0.33 \mu \mathrm{M}$. This is an encouraging result suggesting that RNA binding is indeed the mechanism of Dicer inhibition. Furthermore, the lack of correlation between $K_{D}$ and $\mathrm{IC}_{50}$ values is in accordance with previous reports demonstrating that the affinity is not the only relevant parameter for Dicer inhibition and that binding to a functional site of the targeted RNA is essential for an efficient inhibition activity. ${ }^{13,25}$ It thus happens that strong binders of a particular pre-miRNA are not good inhibitors just because they do not bind a suitable site on the RNA sequence/structure, as is likely the case for PA-4 and PA-5. Interestingly, spermine showed a $K_{\mathrm{D}}$ of $200 \mu \mathrm{M}$, thus showing a 2000 times weaker affinity than PA-3. Azepine and quinolone compounds showed no affinity, further confirming the cooperativity of the two binding domains in the interaction with the RNA target.

The selectivity of binding was also evaluated by measuring $K_{\mathrm{D}}$ in the presence of large excess (100 equiv) of tRNA and DNA, which are abundant intracellular competitors for this kind of RNA ligands. These experiments showed that whereas all the compounds were selective in the presence of tRNA, a slight decrease in affinity was observed in the presence of DNA. More specifically, PA-1, PA-3, and PA-4 retained the affinity for pre-miR-372 even in the presence of an excess

Table 2. Dissociation Constants $\left(K_{\mathrm{D}}, \mu \mathrm{M}\right)$ for the Interaction of pre-miR-372 with PA-1/PA-5

$\begin{array}{ccccc}\text { ID } & K_{\mathrm{D}}{ }^{a} & K_{\mathrm{D}}{ }^{\prime b}(100 \text { equiv tRNA }) & K_{\mathrm{D}}{ }^{\prime} / K_{\mathrm{D}} & K_{\mathrm{D}}{ }^{\prime b}(100 \text { equiv DNA }) \\ \text { PA-1 } & 0.15 \pm 0.01 & 0.14 \pm 0.01 & 1 & 0.17 \pm 0.02 \\ \text { PA-2 } & 0.15 \pm 0.02 & 0.18 \pm 0.03 & 1.1 & 0.32 \pm 0.04 \\ \text { PA-3 } & 0.11 \pm 0.02 & 0.13 \pm 0.02 & 0.1 & 0.18 \pm 0.02 \\ \text { PA-4 } & 0.33 \pm 0.1 & 0.40 \pm 0.09 & 1.0 & 0.62 \pm 0.1 \\ \text { PA-5 } & 0.20 \pm 0.08 & 0.27 \pm 0.1 & 1.9 & 0.57 \pm 0.06\end{array}$

${ }^{a}$ Binding affinities (dissociation constants, $K_{\mathrm{D}}$ ) were evaluated using $10 \mathrm{nM} 5^{\prime}$-FAM-pre-miR-372 beacon in buffer A and represent the average of three independent experiments with their standard deviation. ${ }^{b}$ Selectivity studies were performed in the presence of 10 nM 5 -FAM-pre-miR-372 beacon in buffer A and represent the average of two independent experiments with their standard deviation. 
Table 3. Inhibition Activities $\left(\mathrm{IC}_{50}, \mu \mathrm{M}\right)$ for the Inhibition of miR-373, miR-17, and miR-21 Dicer-Mediated Processing and Corresponding Dissociation Constants $\left(K_{\mathrm{D}}, \mu \mathrm{M}\right)$

\begin{tabular}{|c|c|c|c|c|c|c|c|c|}
\hline ID & $\mathrm{IC}_{50}{ }^{a}$ pre-miR-373 & $K_{\mathrm{D}}{ }^{b}$ pre-miR-373 & $\mathrm{IC}_{50}{ }^{a}$ pre-miR-17 & $K_{\mathrm{D}}^{b}$ pre-miR-17 & $\mathrm{IC}_{50}{ }^{a}$ pre-miR-21 & $K_{\mathrm{D}}^{b}$ pre-miR-21 & $\mathrm{IC}_{50}{ }^{a}$ pre-miR- 155 & $K_{\mathrm{D}}{ }^{b}$ pre-miR- 155 \\
\hline PA-1 & 1.07 & 0.24 & 1.24 & 0.11 & 1.34 & 0.18 & 4.44 & 0.04 \\
\hline PA-2 & 0.29 & 0.20 & 0.80 & 0.13 & 3.76 & 0.15 & 1.5 & 0.09 \\
\hline PA-3 & 0.29 & 0.13 & 0.84 & 0.06 & 1.43 & 0.10 & 1.07 & 0.06 \\
\hline PA-4 & 13.7 & 0.37 & 10.1 & 0.13 & no activity & 0.21 & no activity & 0.18 \\
\hline PA-5 & 12.7 & 0.33 & no activity & 0.11 & no activity & 0.18 & no activity & 0.20 \\
\hline
\end{tabular}

${ }^{a} \mathrm{IC}_{50}$ experiments were performed in the presence of $50 \mathrm{nM}$ of $3^{\prime}$-dabcyl-5'-FAM-pre-miR-372 beacon and $0.25 \mathrm{U}$ of human recombinant Dicer in buffer $\mathrm{A}\left(\mathrm{IC}_{50}\right)$ or $250 \mu \mathrm{g} / \mathrm{mL}$ of MCF-7 lysates $\left(\mathrm{IC}_{50}{ }^{\prime}\right)$ and represent the average of two independent experiments with their standard deviation. ${ }^{b}$ Binding affinities (dissociation constants, $K_{\mathrm{D}}$ ) were evaluated using $10 \mathrm{nM} 5^{\prime}$-FAM-pre-miR-372 beacon in buffer A and represent the average of three independent experiments with their standard deviation. All the values are given with an uncertainty of $\pm 10 \%$.

tRNA and DNA, whereas PA-2 and PA-5 showed $K_{\mathrm{D}}$ values 2 times higher.

In Vitro Selectivity of Compounds PA-1 to PA-5. Further studies about selectivity were performed by measuring the $\mathrm{IC}_{50}$ and $K_{\mathrm{D}}$ values for other pre-miR sequences. So, we chose pre-miR-373, which shares the same seed sequence with miR-372 and originates from the same cluster and pri-miRNA. We also chose to study the activity on oncogenic miRNA-17, which is also highly expressed in gastric cancers tissues and identified to have oncogenic abilities in several other types of cancers. ${ }^{31}$ Then, we screened all compounds on pre-miR-21, which is oncogenic and overexpressed in breast, lung, colon, and prostate cancers but whose upregulation in AGS cells does not affect cellular proliferation. ${ }^{32}$ Finally, miR-155, an oncogenic miRNA not expressed in the same gastric cancers as miR-372 and miR-373 but involved in normal immune function, was employed in this selectivity study as unrelated to the previously described miRNAs. ${ }^{33}$

These experiments showed that the studied polyamines can bind and inhibit the processing of pre-miRNAs different from pre-miR-372 but with various selectivity profiles. First, the comparison of $\mathrm{IC}_{50}$ and $K_{\mathrm{D}}$ between pre-miR-372 and premiR-373 (Table 3) showed that the most efficient inhibitors and ligands of pre-miR-372 (PA-1, PA-2, and PA-3) have the same or even better $\mathrm{IC}_{50}$ and $K_{\mathrm{D}}$ values for pre-miR-373. This could of course be useful in intracellular assays performed on AGS cells because both are involved in cancer cells proliferation. The comparison of these values with the other pre-miRNAs (pre-miR-17, pre-miR-21, and pre-miR-155) led to the observation that PA-1 maintained both activity and affinity for pre-miR-21 and pre-miR-17, whereas it bore a 4times lower activity for pre-miR-155. More interestingly, PA-2 and PA-3 lost the activity for these three pre-miRNAs with $\mathrm{IC}_{50}$ from 2 to 10 times higher.

Because the affinities are very similarly independent of the considered pre-miRNA, it appears that the mode of binding and the site of the interaction, which are certainly different in the five studied pre-miRNAs, have a pivotal role in the inhibition efficiency and determine the selectivity profile. PA-3 shows the best selectivity in all the performed assays because (i) it is the most selective inhibitor of pre-miR-372 processing in the presence of cell lysates, (ii) it is the most selective binder of pre-miR-372 in the presence of a large excess of competitors (tRNA and DNA), and (iii) it is selective for pre-miR-372 and pre-miR-373 when its activity and affinity are compared to the ones toward other pre-miRNAs. Thus, all the compounds are very good RNA binders, but only PA-1, PA-2, and PA-3 also showed selectivity with respect to tRNA, DNA, and other premiRNAs. PA-3 also showed the best selectivity in the inhibition assays and thus appears as the most promising pre-miR-372 ligand and inhibitor.

Mode of Binding of PA-3 with miR-372. PA-3 is an efficient binder of pre-miR-372 and a selective inhibitor of its Dicer-mediated processing, so we decided to explore its molecular mechanism of binding. As a first attempt to better understand its efficacy, we decided to measure the thermodynamic parameters of the interaction formed with pre-miR-372 in comparison with the other polyamines. Free energies of Gibbs $\left(\Delta G^{\circ}\right)$ were first calculated from the dissociation constants $\left(\Delta G^{\circ}=-R T \ln K_{\mathrm{D}}\right)$ for all compounds and found to be very similar (from -35 to $-45 \mathrm{~kJ} / \mathrm{mol}$ ), with PA-3 forming the most favorable interaction complex (Figure 3). To

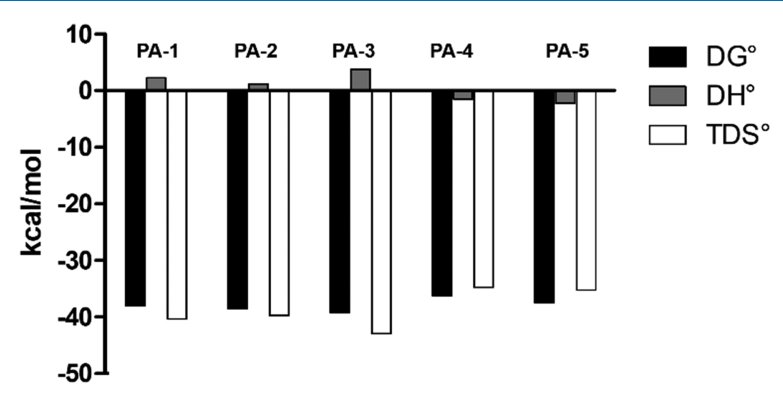

Figure 3. Thermodynamic profiles for the high-affinity binding of heterocycle-spermine conjugates PA-1 to PA-5 to the pre-miR-372 at $20{ }^{\circ} \mathrm{C}: \Delta G^{\circ}$ (black), $\Delta H^{\circ}$ (gray), and $-T \Delta S^{\circ}$ (white).

complete the thermodynamic binding profiles, the enthalpic $\left(\Delta H^{\circ}\right)$ and the entropic $\left(-T \Delta S^{\circ}\right)$ energy contributions were determined after the determination of $\Delta G^{\circ}{ }_{T}$ at several temperatures $(278-308 \mathrm{~K})$. The results of these thermodynamic analysis are summarized in Figure 3. In all the cases, the formation of PAs/pre-miR-372 complexes is driven by entropy, suggesting that most of the formed interactions are strong but not specific and indicate a strong desolvation effect occurring during interaction. Noteworthy, the most efficient inhibitors, PA-1, PA-2, and PA-3, bear a slightly positive enthalpy factor, suggesting that the binding affinity and especially selectivity could be greatly improved upon formation of further noncovalent interactions such as H-bonds, $\pi$ stacking, $\pi$-ions, and, in general, all nonelectrostatic interactions that contribute to a more favorable enthalpy of interactions. These results further underline the potential of this kind of compounds in the development of strong and selective inhibitors.

On the basis of the knowledge acquired during these thermodynamic studies, we performed molecular docking studies for compound PA-3. As illustrated in Figure 4, PA-3 


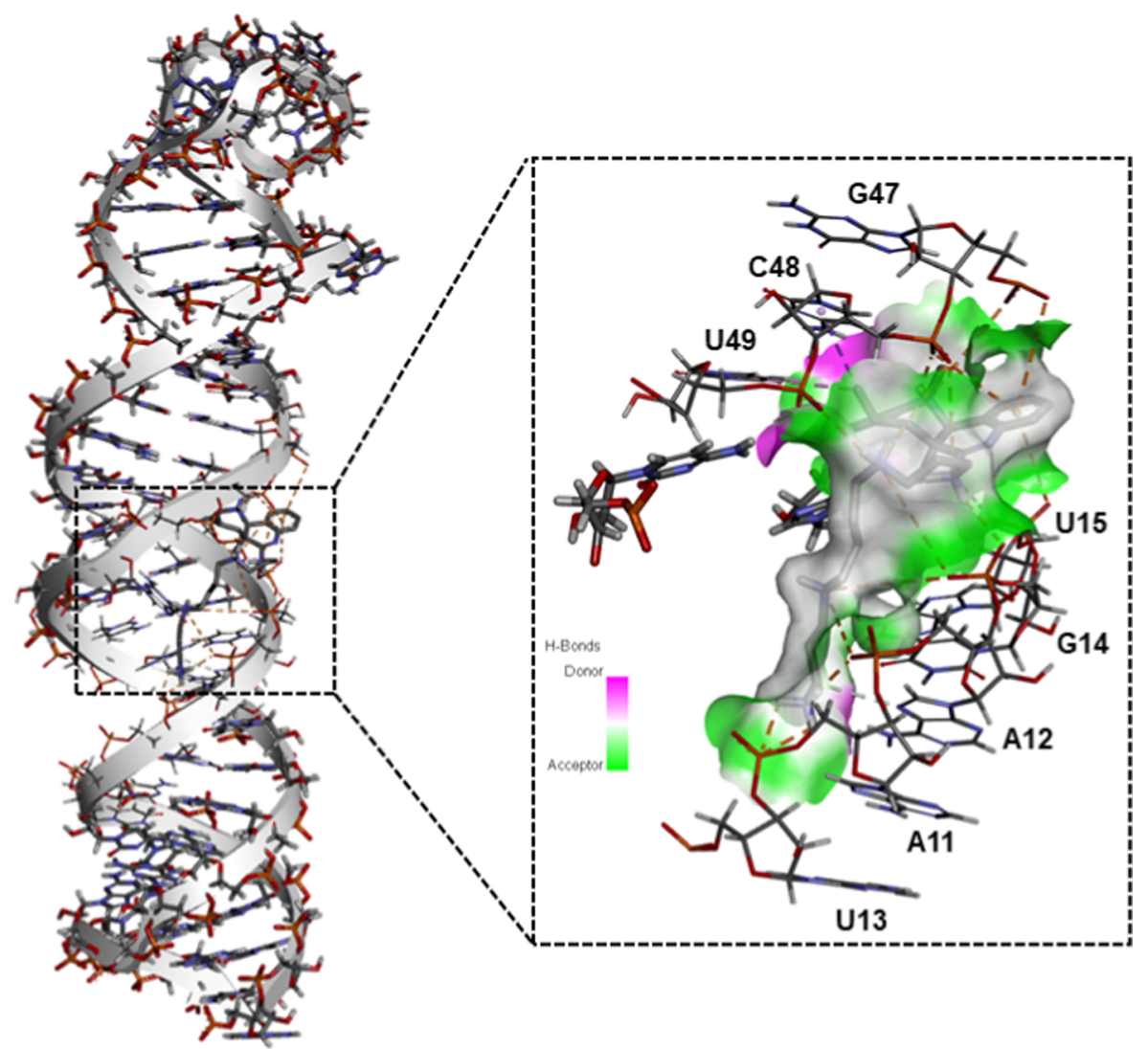

Figure 4. Molecular docking of PA-3 interaction with pre-miR-372 hairpin loop performed using autodock 4 wherein the grid boxes were fixed on the entire RNA sequence.

likely binds close to the internal G-loop (residues A11-G14 and G47-U49), but with a better fit of the binding pocket compared to PA-1. Furthermore, all compound moieties, i.e., the polyamine side chain and the heterocyclic group, are involved in the formation of the complex (Figure S3). Although it is known that spermine interacts nonspecifically with various biologically relevant $\mathrm{RNAs}^{34}$ the heterocyclic moiety probably brings selective interactions. As we previously demonstrated, binding close to the G16-G52 internal loop is particularly favorable for the inhibition of Dicer-mediated processing because even if these residues are not located at the catalytic site, they are essential for correct RNA recognition by Dicer enzyme. Binding of an efficient RNA ligand to the sequence that will constitute a mature miRNA duplex likely induces changes in the conformation of the pre-miRNA, thus inhibiting Dicer recognition and binding to this structure. As a confirmation, PA-5, which is a good RNA ligand but not able to inhibit Dicer processing, binds to a different site in the lower stem region (C7-A10 and C54-U57), a site that is likely less favorable for inhibition (Figure S4 in the Supporting Information). Finally, most of the formed interactions are represented by $\pi$-anion interactions, $\pi$-stacking, and electrostatic interactions, further highlighting that the formation of more specific $\mathrm{H}$-bonds would undoubtedly increase the affinity and especially the selectivity. PA-3 confirms to be a very promising compound for future intracellular studies, but it also opens the way for the design of chemically modified analogues with better activity and selectivity features.

\section{CONCLUSIONS}

In conclusion, the evaluation of various heterocycle-spermine conjugates analogues of PA-1, a previously identified polyamine that was able to selectively inhibit miRNA biogenesis in vitro and in cells, allowed for the characterization of the selectivity profiles and modes of binding for these compounds. PA-3 emerged as the most promising compound bearing strong affinity, submicromolar inhibition activity, and selectivity for the targeted RNA. This compound bears an extended and constrained heterocyclic and aromatic substituent conjugated to a spermine side chain. The detailed study of the mode of binding revealed that the enthalpic component of the free energy of binding should be improved to increase the affinity and especially the selectivity. Thus, the introduction of heteroatoms and substituents that could increase the number of specific interactions such as $\mathrm{H}$-bonds would increase the enthalpy of the interaction and eventually specificity and inhibition efficacy. This study further demonstrates that functionalized polyamines are promising compounds for the interference with oncogenic miRNAs biogenesis and opens the way for future modifications of these compounds both on the heterocyclic moiety and on the spermine residue toward their intracellular applications. We thus plan to further modify the chemical structure of PA-1 and PA-3 to reach these additional sites of interaction and improve binding and inhibition.

\section{MATERIALS AND METHODS}

Chemical synthesis and characterization of compounds PA-1 to PA-5 have been previously reported. ${ }^{30}$ 
Biochemical Studies and RNA. Solutions and buffers employed in $\mathrm{IC}_{50}$ assays and $K_{\mathrm{D}}$ measurements were all filtered through $0.22 \mu \mathrm{m}$ Millipore filters (GP ExpressPLUS membrane).

RNA and DNA oligonucleotides were purchased from IBA $\mathrm{GmbH}$ (Goettingen, Germany). A mixture of yeast pre- and mature tRNAs (containing $>30$ different species) was purchased from Sigma (type X-SA). Human recombinant Dicer enzyme was purchased from Genlantis at $0.5 \mathrm{U} / \mu \mathrm{L}$. Tris. $\mathrm{HCl} 20 \mathrm{mM}$, pH 7.4 containing $12 \mathrm{mM} \mathrm{NaCl}, 3 \mathrm{mM} \mathrm{MgCl}$, and $1 \mathrm{mM}$ dithiothreitol, was used for FRET assays and $K_{\mathrm{D}}$ determination (buffer A).

Fluorescence-Based Assays. The $\mathrm{IC}_{50}$ assays and $K_{\mathrm{D}}$ determinations were performed using a 5070 EpMotion automated pipetting system (Eppendorf) in a final volume of 40 and $60 \mu \mathrm{L}$, respectively, and in 384-well black plates (Greiner bio-one). Each experiment was performed in duplicate and repeated three times. RNA (5'-FAM-pre-miR$372-3^{\prime}$-DAB for FRET experiments and 5'-FAM-pre-miR-372 for $K_{\mathrm{D}}$ measurements) was folded using a thermocycler (ThermoStatPlus Eppendorf) as follows: each RNA was diluted in $1 \mathrm{~mL}$ of buffer $\mathrm{A}$, heated at $90{ }^{\circ} \mathrm{C}$ for $2 \mathrm{~min}$, then cooled to $4{ }^{\circ} \mathrm{C}$ for $10 \mathrm{~min}$, and finally incubated at $25{ }^{\circ} \mathrm{C}$ for $15 \mathrm{~min}$. After refolding, the RNA was diluted to a working concentration of $100 \mathrm{nM}$ (for FRET-Dicer assays) or $10 \mathrm{nM}$ (for binding assays) through addition of the appropriate amount of buffer A. During $\mathrm{IC}_{50}$ measurements, $20 \mu \mathrm{L}$ of the pre-miRNA solution were added in each well containing $10 \mu \mathrm{L}$ of each desired ligand concentration. These reaction mixtures were incubated at room temperature for $30 \mathrm{~min}$. Human recombinant Dicer $(0.25 \mathrm{U})$ diluted in $10 \mu \mathrm{L}$ or $10 \mu \mathrm{L}$ of MCF-7 cells lysates $(250 \mu \mathrm{g} / \mathrm{mL})$ were then added to a final volume of $40 \mu \mathrm{L}$, and the fluorescence measured after $4 \mathrm{~h}$. Each ligand was added in 12 different dilutions (from 0.244 to $500 \mu \mathrm{M}$ ) during $\mathrm{IC}_{50}$ experiments. During binding assays, 30 $\mu \mathrm{L}$ of the pre-miRNA were added to $30 \mu \mathrm{L}$ of each desired ligand concentration to reach a final volume of $60 \mu \mathrm{L}$. Each ligand was added in 15 different dilutions (from 61 to $1 \mathrm{mM}$ ) and fluorescence was measured after incubating the plate overnight. Fluorescence measurements were performed on a GeniosPro (Tecan) with an excitation filter of $485 \pm 10 \mathrm{~nm}$ and an emission filter of $535 \pm 15 \mathrm{~nm}$. Each point was measured 10 times with a $500 \mu$ s integration time and averaged. Inhibition data were analyzed using Graphpad Prism 5 software using a nonlinear regression following the equation: $Y=$ bottom $+($ top - bottom $) /\left(1+10^{\left[\left(\log \mathrm{IC}_{50}-X\right) \times \text { Hills slope }\right]}\right) . \mathrm{A}$ one-to-one stoichiometry model was used to fit the binding profiles.

For competitive experiments in the presence of a dsDNA, a 15-mer sequence ( $5^{\prime}$-CGTTTT TATTTTTGC-3') and its complement, annealed beforehand, were added to buffer $\mathrm{A}$ to obtain a 100-fold nucleotide excess regarding pre-miR-372 (448 nM duplex; 10 nM RNA).

For competitive experiments in the presence of a tRNA, a mixture of pre- and mature yeast tRNAs (containing over 30 different species from baker's yeast (Saccharomyces cerevisiae, Sigma, type X-SA)) was added to buffer A to obtain a 100 -fold nucleotide excess regarding pre-miR-372 (1.13 $\mu \mathrm{M}$ tRNA; 10 nM RNA). Stock solutions of tRNA were prepared in water and quantified using an extinction coefficient of $9640 \mathrm{~cm}^{-1}$ $\mathrm{M}^{-1}$ per base.

To check that the fluorescence quenching was not a result of nonspecific interactions between PA compounds and the fluorophore, titrations of a $5 \mathrm{nM}$ solution of a fluorescein with PA-1 to PA-5 ranging from 0.01 to $1500 \mu \mathrm{M}$ were carried out. No significant changes in the fluorescence intensity was observed (less than $10 \%$, data not shown), thus confirming that the observed fluorescence decreases very likely reflect the binding of PA-1 to PA-5 compounds to the labeled premiRNA fragment rather than nonspecific interactions with the fluorophore.

\section{DATA ANALYSIS}

Inhibition data ( $\mathrm{IC}_{50}$ FRET experiments) and binding data $\left(K_{\mathrm{D}}\right.$ experiments) were analyzed using Prism 5 (GraphPad Software) by nonlinear regression following the equation

$$
\begin{aligned}
Y= & \text { bottom }+(\text { top }- \text { bottom }) \\
& /\left(1+10^{\left[\left(\log \mathrm{IC}_{50}-X\right) \times \text { Hill slope }\right]}\right)
\end{aligned}
$$

$K_{\mathrm{D}}$ values were converted to $\Delta G^{\circ}$ values as $\Delta G^{\circ}=-R T \ln K_{\mathrm{D}}$.

For thermodynamic analysis, $\Delta G^{\circ}$ values were plotted versus temperature $(T)$. Nonlinear regression using the threeparameter fit in Prism 5 was used to fit the following equation to the data

$$
\begin{aligned}
\Delta G^{\circ} T= & \Delta H_{T_{\mathrm{r}}}^{\circ}+\Delta C_{\mathrm{p}}\left(T-T_{\mathrm{r}}\right)-T \Delta S_{T_{\mathrm{r}}}^{\circ} \\
& -T \Delta C_{\mathrm{p}} \ln \left(T / T_{\mathrm{r}}\right)
\end{aligned}
$$

where $T_{\mathrm{r}}$ is a constant reference temperature (in our study $T_{\mathrm{r}}=$ 293.15 K) and the three fit parameters are as follows: $\Delta H^{\circ}{ }_{T_{r}}$, the change in enthalpy on binding at $T_{\mathrm{r}} ; \Delta S_{T_{\mathrm{r}}}^{\circ}$, the change in entropy on binding at $T_{\mathrm{r}}$; and $\Delta C_{\mathrm{p}}$, the change in heat capacity. $\Delta C_{p}$ was assumed to be independent of temperature; inclusion of a $\Delta C_{\mathrm{p}} / \Delta T$ term in the analysis did not improve the quality of the fits and gave larger standard errors for the returned parameters.

$\Delta H^{\circ}{ }_{T}$ and $\Delta S^{\circ}{ }_{T}$ were calculated based on the correlation between $\Delta G^{\circ}$ values and $T$ using the following equation

$$
\Delta H_{T}^{\circ}=\Delta H_{T_{\mathrm{r}}}^{\circ}+\Delta C_{\mathrm{p}}\left(T-T_{\mathrm{r}}\right)
$$

and

$$
\Delta S_{T}^{\circ}=\Delta S_{T_{\mathrm{r}}}^{\circ}+\Delta C_{\mathrm{p}} \ln \left(T / T_{\mathrm{r}}\right)
$$

where $\Delta H^{\circ}{ }_{T}$ is the change in enthalpy on binding at $T\left(25^{\circ} \mathrm{C}\right)$ and $\Delta S^{\circ}{ }_{T}$ is the change in entropy on binding at $T\left(25^{\circ} \mathrm{C}\right)$.

Molecular Modeling and Docking. Molecular modeling was performed first using the MC-Fold/MC-Sym pipeline (http://www.major.iric.ca/MC-Pipeline/) to predict RNA secondary and tertiary structure of the studied pre-miRNAs. The MC-Fold/MC-Sym pipeline is based on the upload of the RNA sequence to MC-Fold, which then outputs the secondary structure. This latter is then directly input to MC-Sym, which leads to tertiary structures. Pre-miRNA sequences were obtained from the miRBase database (http://www.mirbase. org/). The TINKER Molecular Modeling Package (http:// dasher.wustl.edu/tinker/) was employed to perform energy optimization on the 3D model.

For docking with AutoDock, polar hydrogen atoms, Kollman united charges, and solvent parameters were applied to the RNA using pmol2q script (http://www.sourcefiles.org/ Scientific/Biology/Proteins/pmol2q 2.3.0.tar.gz). ${ }^{35}$ This script converts the .pdb file format of the RNA template to the .pdbqt file format, which is compatible with AutoDock program version 4 (http://autodock.scripps.edu/). AutoDock 
program version 4 was used for the molecular docking of premiR-372/PA-3. The rotational bonds of the ligand were treated as flexible, whereas the receptor was kept rigid. Grid box was fixed including the entire RNA sequence. RNA-ligand interactions were analyzed and visualized using Discovery Studio Visualizer version 4.1 (http://accelrys.com/products/ discovery-studio/).

\section{ASSOCIATED CONTENT}

\section{S Supporting Information}

The Supporting Information is available free of charge on the ACS Publications website at DOI: 10.1021/acsomega.8b02681.

Oligonucleotide sequences, binding and inhibition curves, molecular docking of PA-3 and PA-5 (PDF)

\section{AUTHOR INFORMATION}

\section{Corresponding Author}

*E-mail: maria.duca@univ-cotedazur.fr. Tel: +33-4-92076153. Fax: +33-4-92076121.

\section{ORCID $\odot$}

Maria Duca: 0000-0002-2666-6180

Notes

The authors declare no competing financial interest.

\section{ACKNOWLEDGMENTS}

This work was supported by two grants from Agence Nationale de la Recherche (ANR-11-JS07-011-01 and ANR-14-CE210006). We would like to thank Dr. Annie Ladoux for kindly providing us MCF-7 lysates and the "GIS Chimiothèque Nationale" for the constitution of the French National Chemical Library and the Essential Chemical Library whose screening allowed for the identification of polyamines as promising inhibitors of miRNAs biogenesis.

\section{REFERENCES}

(1) Eddy, S. R. Non-coding RNA genes and the modern RNA world. Nat. Rev. Genet. 2001, 2, 919-929.

(2) Cech, T. R.; Steitz, J. A. The noncoding RNA revolution-trashing old rules to forge new ones. Cell 2014, 157, 77-94.

(3) Ambros, V. The evolution of our thinking about microRNAs. Nat. Med. 2008, 14, 1036-1040.

(4) Bartel, D. P. MicroRNAs: target recognition and regulatory functions. Cell 2009, 136, 215-233.

(5) Kim, V. N.; Han, J.; Siomi, M. C. Biogenesis of small RNAs in animals. Nat. Rev. Mol. Cell Biol. 2009, 10, 126-139.

(6) MacRae, I. J.; Ma, E.; Zhou, M.; Robinson, C. V.; Doudna, J. A. In vitro reconstitution of the human RISC-loading complex. Proc. Natl. Acad. Sci. U.S.A. 2008, 105, 512-517.

(7) Iorio, M. V.; Croce, C. M. microRNA involvement in human cancer. Carcinogenesis 2012, 33, 1126-1133.

(8) Li, Z.; Rana, T. M. Therapeutic targeting of microRNAs: current status and future challenges. Nat. Rev. Drug Discovery 2014, 13, 622638.

(9) Ling, H.; Fabbri, M.; Calin, G. A. MicroRNAs and other noncoding RNAs as targets for anticancer drug development. Nat. Rev. Drug Discovery 2013, 12, 847-865.

(10) Warner, K. D.; Hajdin, C. E.; Weeks, K. M. Principles for targeting RNA with drug-like small molecules. Nat. Rev. Drug Discovery 2018, 17, 547-558.

(11) Velagapudi, S. P.; Vummidi, B. R.; Disney, M. D. Small molecule chemical probes of microRNA function. Curr. Opin. Chem. Biol. 2015, 24, 97-103.
(12) Childs-Disney, J. L.; Disney, M. D. Small Molecule Targeting of a MicroRNA Associated with Hepatocellular Carcinoma. ACS Chem. Biol. 2016, 11, 375-380.

(13) Costales, M. G.; Haga, C. L.; Velagapudi, S. P.; Childs-Disney, J. L.; Phinney, D. G.; Disney, M. D. Small Molecule Inhibition of microRNA-210 Reprograms an Oncogenic Hypoxic Circuit. J. Am. Chem. Soc. 2017, 139, 3446-3455.

(14) Gumireddy, K.; Young, D. D.; Xiong, X.; Hogenesch, J. B.; Huang, Q.; Deiters, A. Small-molecule inhibitors of microrna miR-21 function. Angew. Chem., Int. Ed. 2008, 47, 7482-7484.

(15) Haga, C. L.; Velagapudi, S. P.; Strivelli, J. R.; Yang, W. Y.; Disney, M. D.; Phinney, D. G. Small Molecule Inhibition of miR-544 Biogenesis Disrupts Adaptive Responses to Hypoxia by Modulating ATM-mTOR Signaling. ACS Chem. Biol. 2015, 10, 2267-2276.

(16) Shi, Z.; Zhang, J.; Qian, X.; Han, L.; Zhang, K.; Chen, L.; Liu, J.; Ren, Y.; Yang, M.; Zhang, A.; Pu, P.; Kang, C. AC1MMYR2, an inhibitor of dicer-mediated biogenesis of Oncomir miR-21, reverses epithelial-mesenchymal transition and suppresses tumor growth and progression. Cancer Res. 2013, 73, 5519-5531.

(17) Velagapudi, S. P.; Gallo, S. M.; Disney, M. D. Sequence-based design of bioactive small molecules that target precursor microRNAs. Nat. Chem. Biol. 2014, 10, 291-297.

(18) Vo, D. D.; Staedel, C.; Zehnacker, L.; Benhida, R.; Darfeuille, F.; Duca, M. Targeting the production of oncogenic microRNAs with multimodal synthetic small molecules. ACS Chem. Biol. 2014, 9, 711721.

(19) Vo, D. D.; Tran, T. P.; Staedel, C.; Benhida, R.; Darfeuille, F.; Di Giorgio, A.; Duca, M. Oncogenic MicroRNAs Biogenesis as a Drug Target: Structure-Activity Relationship Studies on New Aminoglycoside Conjugates. Chemistry 2016, 22, 5350-5362.

(20) Staedel, C.; Tran, T. P. A.; Giraud, J.; Darfeuille, F.; Di Giorgio, A.; Tourasse, N. J.; Salin, F.; Uriac, P.; Duca, M. Modulation of oncogenic miRNA biogenesis using functionalized polyamines. Sci. Rep. 2018, 8, No. 1667.

(21) Velagapudi, S. P.; Cameron, M. D.; Haga, C. L.; Rosenberg, L. H.; Lafitte, M.; Duckett, D. R.; Phinney, D. G.; Disney, M. D. Design of a small molecule against an oncogenic noncoding RNA. Proc. Natl. Acad. Sci. U.S.A. 2016, 113, 5898-5903.

(22) Di Giorgio, A.; Tran, T. P.; Duca, M. Small-molecule approaches toward the targeting of oncogenic miRNAs: roadmap for the discovery of RNA modulators. Future Med. Chem. 2016, 8, $803-816$.

(23) Disney, M. D.; Winkelsas, A. M.; Velagapudi, S. P.; Southern, M.; Fallahi, M.; Childs-Disney, J. L. Inforna 2.0: A Platform for the Sequence-Based Design of Small Molecules Targeting Structured RNAs. ACS Chem. Biol. 2016, 11, 1720-1728.

(24) Monroig, P. C.; Chen, L.; Zhang, S.; Calin, G. A. Small molecule compounds targeting miRNAs for cancer therapy. Adv. Drug Delivery Rev. 2015, 81, 104-116.

(25) Tran, T. P.; Vo, D. D.; Di Giorgio, A.; Duca, M. Ribosometargeting antibiotics as inhibitors of oncogenic microRNAs biogenesis: Old scaffolds for new perspectives in RNA targeting. Bioorg. Med. Chem. 2015, 23, 5334-5344.

(26) Velagapudi, S. P.; Disney, M. D. Two-dimensional combinatorial screening enables the bottom-up design of a microRNA-10b inhibitor. Chem. Commun. 2014, 50, 3027-3029.

(27) Velagapudi, S. P.; Seedhouse, S. J.; Disney, M. D. Structureactivity relationships through sequencing (StARTS) defines optimal and suboptimal RNA motif targets for small molecules. Angew. Chem., Int. Ed. 2010, 49, 3816-3818.

(28) Voorhoeve, P. M.; le Sage, C.; Schrier, M.; Gillis, A. J.; Stoop, H.; Nagel, R.; Liu, Y. P.; van Duijse, J.; Drost, J.; Griekspoor, A.; Zlotorynski, E.; Yabuta, N.; De Vita, G.; Nojima, H.; Looijenga, L. H.; Agami, R. A genetic screen implicates miRNA-372 and miRNA-373 as oncogenes in testicular germ cell tumors. Cell 2006, 124, 1169-1181.

(29) Lee, K. H.; Goan, Y. G.; Hsiao, M.; Lee, C. H.; Jian, S. H.; Lin, J. T.; Chen, Y. L.; Lu, P. J. MicroRNA-373 (miR-373) posttranscriptionally regulates large tumor suppressor, homolog 2 
(LATS2) and stimulates proliferation in human esophageal cancer. Exp. Cell Res. 2009, 315, 2529-2538.

(30) Delcros, J. G.; Tomasi, S.; Duhieu, S.; Foucault, M.; Martin, B.; Le Roch, M.; Eifler-Lima, V.; Renault, J.; Uriac, P. Effect of polyamine homologation on the transport and biological properties of heterocyclic amidines. J. Med. Chem. 2006, 49, 232-245.

(31) Krichevsky, A. M.; Gabriely, G. miR-21: a small multi-faceted RNA. J. Cell. Mol. Med. 2009, 13, 39-53.

(32) Wu, Q.; Luo, G.; Yang, Z.; Zhu, F.; An, Y.; Shi, Y.; Fan, D. miR$17-5 p$ promotes proliferation by targeting SOCS6 in gastric cancer cells. FEBS Lett. 2014, 588, 2055-2062.

(33) Costinean, S.; Zanesi, N.; Pekarsky, Y.; Tili, E.; Volinia, S.; Heerema, N.; Croce, C. M. Pre-B cell proliferation and lymphoblastic leukemia/high-grade lymphoma in $\mathrm{E}(\mathrm{mu})$-miR155 transgenic mice. Proc. Natl. Acad. Sci. U.S.A. 2006, 103, 7024-7029.

(34) Lightfoot, H. L.; Hall, J. Endogenous polyamine function-the RNA perspective. Nucleic Acids Res. 2014, 42, 11275-11290.

(35) Morris, G. M.; Huey, R.; Lindstrom, W.; Sanner, M. F.; Belew, R. K.; Goodsell, D. S.; Olson, A. J. AutoDock4 and AutoDockTools4: Automated docking with selective receptor flexibility. J. Comput. Chem. 2009, 30, 2785-2791. 